\title{
Digitalisierungsprojekte der WLB ${ }^{1}$
}

Die Württembergische Landesbibliothek betreibt im Rahmen des 2011 begonnenen Landesdigitalisierungsprogramms eine systematische Digitalisierung wichtiger Bestandsgruppen. Das Motto dieses aus Mitteln des Ministeriums für Wissenschaft, Forschung und Kunst Baden-Württemberg finanzierten Projekts wurde von der Formel "Vom Tresor in die Welt" in das weiter gefasste Programm „Dokumentenerbe digital - Digitalisierung historischer Bestände baden-württembergischer Bibliotheken" umbenannt. Dies deutet auf eine Ausweitung in Frage kommender Bestände bzw. eine Modifikation der Auswahlkriterien hin. Zunächst ging es um die wertvollsten bzw. fragilsten Bestandsgruppen, um Zimelien und für die Benutzung gesperrte Bestände. Dann rückte der Regionalbezug als Auswahlkriterium verstärkt in das Blickfeld, auch im Zusammenhang mit dem Portal LEO-BW (Landeskunde entdecken online Baden-Württemberg). Deutlich war aber auch schon hier, dass die Tatsache der Aufbewahrung in einer Bibliothek Baden-Württembergs ein Stück als regionales Kulturgut auszeichnet, auch wenn kein direkter formaler oder inhaltlicher Landesbezug besteht. Mit der Umbenennung wird dieser Akzent dahingehend verstärkt, dass historische Bestände in ihrem gewachsenen Profil als Dokumentenerbe verstanden werden. Dieses gilt es durch Digitalisierung zu schützen und zugleich elektronisch verfügbar zu machen.

Das Landesdigitalisierungsprogramm ist den von der Deutschen Forschungsgemeinschaft (DFG) für die Förderungswürdigkeit zur Digitalisierung (2016) genannten Kriterien "der Forschungsrelevanz oder der wissenschaftlichen Nachfrage " verpflichtet. Dazu können geschlossene Sammlungen, Einzelstücke mit ausgaben- oder exemplarspezifischer Rarität, aber auch neuere Titel des 19. und frühen 20. Jahrhunderts gehören, sofern sie für die Forschung sonst schwer zugänglich oder aber stark nachgefragt sind. Die Definition der Einzelprojekte hat dabei die Bestände der Historischen Sammlungen in ihrer Breite und Vielfalt zu berücksichtigen.
Ende Juli 2019 sind über die Digitalen Sammlungen der WLB 2.065.942 Bilder aus 14.021 bibliographischen Einheiten zugänglich (Stand: 24.7.2019).

Ein Auswahlkriterium zur Priorisierung besonders digitalisierungswürdiger Bestände ist deren Unikalität aufgrund bestimmter Ausstattungsmerkmale. Dazu zählen vor allem Drucke mit kolorierten Holzschnitten bzw. durch Buchmalerei eingebrachten Illustrationen (z.B. Initialen in Deckfarbenmalerei).

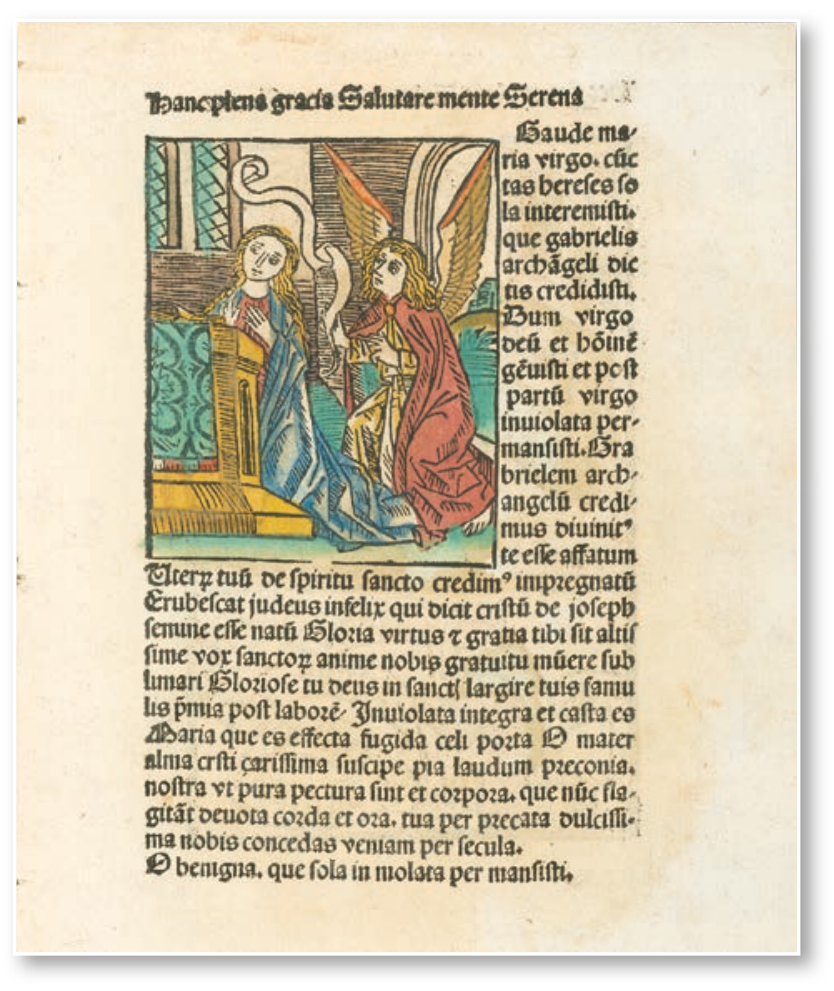

Abb. 1: Kolorierte Inkunabel (Inc.qt.6086)

So steht ein Teilprojekt zur Digitalisierung von knapp 80 kolorierten Inkunabeln vor dem Abschluss. Die frühen Holzschnitte waren mit ihrer konturenartigen Darstellungsweise auf spätere Kolorierung angelegt. Neben Bibelausgaben, liturgischen und juristischen Büchern versprachen insbesondere handliche und illustrierte Bände mit frömmigkeitsspezifischen Themen eine hohe Akzeptanz. Als Beispiel mag hier die ca. 1487/1488 in Basel gedruckte Ausgabe des "Defensorium inviolatae virginitatis Mariae" (GW 10275) des Dominikaners Franciscus de Retza (ca. 1343-1427) dienen (Inc.qt.6086). Charakteristisch für die spätmittelalterliche Gesellschaft war eine intensive marianische Volksfrömmigkeit in Verbindung 
mit dem Wallfahrtswesen. Marias Lebensstationen wurden mit Holzschnitten veranschaulicht. Außerdem wurden Inkunabeln mit unikalem WLBBesitz sowie aus dem Vorbesitz württembergischer Herrscher digitalisiert.

Noch im Gang ist die Digitalisierung kolorierter, großformatiger Pflanzenbücher, überwiegend aus dem 18. und 19. Jahrhundert. 73 bibliographische Einheiten sind bereits online. Meist handelt es sich um Bildbände mit kolorierten Kupferstichen oder

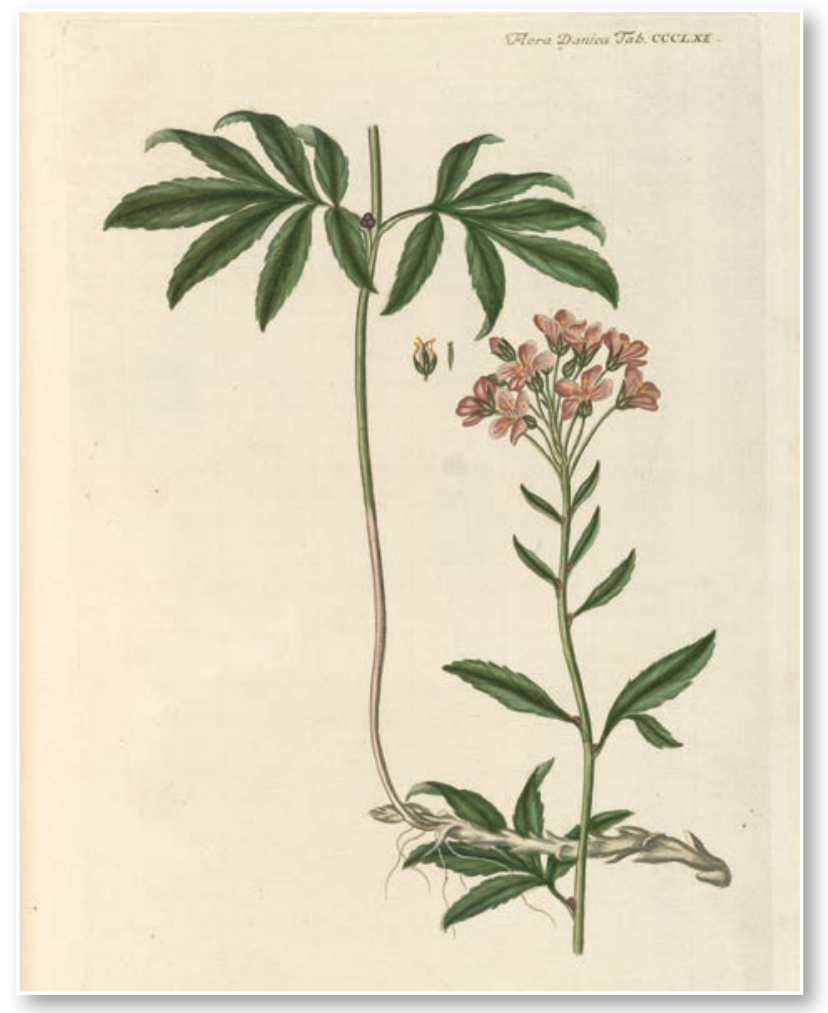

Abb. 2: Koloriertes Pflanzenbuch (Nat.G.fol.147-3)

Naturselbstdrucken. Als Beispiel sei hier das fünfzehnbändige Werk „Flora Danica“, Kopenhagen 1766-1861, genannt (Nat.G.fol.147). Die WappenSupralibros-Einbände der ersten fünf Bände deuten darauf hin, dass Herzog Carl Eugen sie bei seiner Reise nach Kopenhagen 1784 vom dänischen König Christian VII. (1749-1808) geschenkt erhielt.

Im Jahr 2018 konnte die Digitalisierung von über 900 Einblattdrucken des 16. bis 19. Jahrhunderts abgeschlossen werden. Dabei handelt es sich häufig um amtliche Verlautbarungen mit Regionalbezug. Begonnen wurde mit der Digitalisierung von ca. 80 raren bzw. unikalen Drucken des 16. Jahrhunderts. Dies knüpft an ein kooperatives Projekt zur Digitalisierung der im deutschen Sprachraum im 16. Jahrhundert gedruckten Werke (VD16) an, bei dem die WLB etwa 600 bibliographische Einheiten beisteuern konnte. Dabei kam es auch zur Entdeckung bisher im VD16 noch nicht bekannter bzw. vermerkter Ausgaben.

Wegen ihrer historischen Bedeutung und Seltenheit von besonderer Forschungsrelevanz sind die frühen Polyglottenbibeln. 11 der 33 Einheiten der ersten Projektphase stehen bereits online zur Verfügung. Dazu zählt auch die Complutensische Polyglotte, die 1514 in sechs Bänden erschien (Bb polygl.1514 01) und von Martin Luther für seine

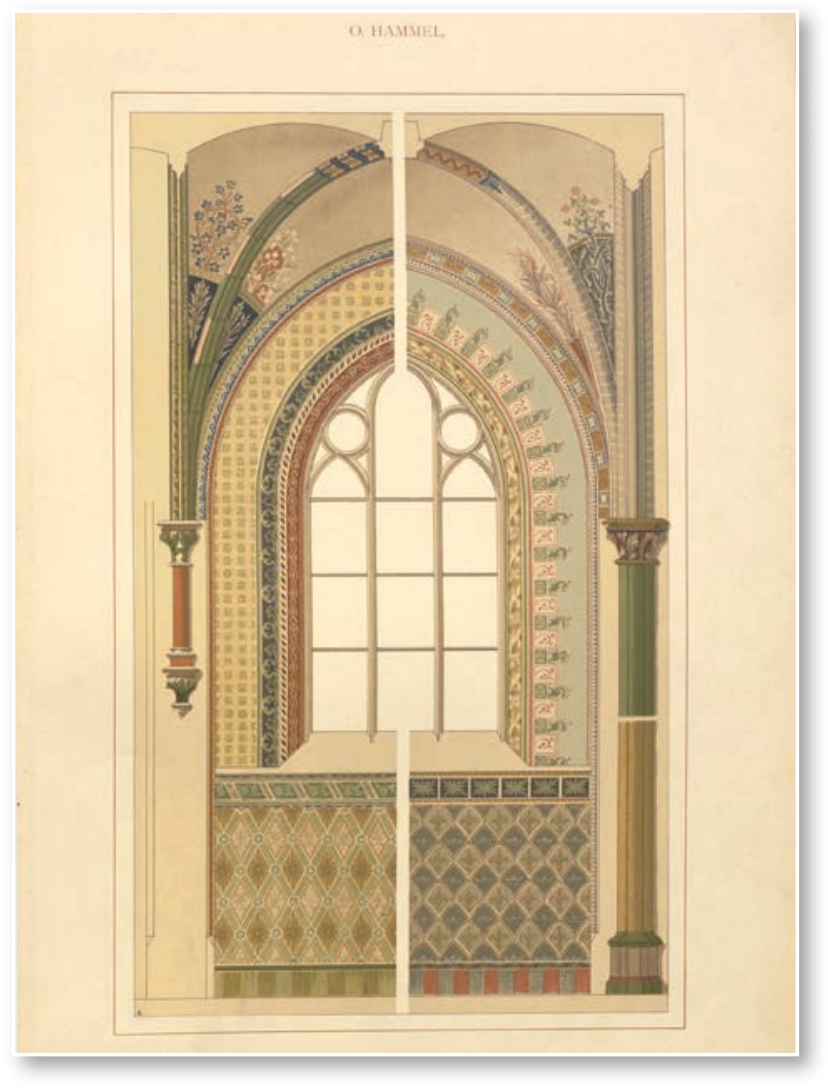

Abb. 3: Landesgewerbeamts-Band zur Innenarchitektur (A22g/346)

Bibelübersetzung mit herangezogen wurde. Überwiegend im Zeitraum zwischen 1870 und 1920 erschienen sind die meist großformatigen Bildbände aus dem Vorbesitz der Bibliothek für Technik, Kunst und Wirtschaft des Landesgewerbeamts Stuttgart. Darin sind häufig Illustrationen zu herausragenden Beispielen der Architektur, des Handwerks und der industriellen Produktion mit kurzen textlichen Erläuterungen enthalten. Im Juli 2019 waren 241 Titel elektronisch verfügbar. Sie wurden größtenteils mit Hilfe einer Dienstleisterfirma digitalisiert und anschließend in der WLB 
katalogisiert. Bis zum Jahresende 2019 werden über 200 weitere Ausgaben hinzukommen.

Eine ergiebige Primärquelle für die interdisziplinäre Forschung sind die Gelegenheitsschriften, die v.a. anlässlich von Beerdigungen und Hochzeiten gedruckt wurden (Signaturgruppe: Fam.Pr.). Sie enthalten neben theologischen Abhandlungen meist detaillierte Lebensläufe, auch mit Angaben zu Krankheiten, sowie lyrische Beiträge und Illustrationen zum Todesmotiv. Von der bisher überwiegend noch nicht katalogisierten, ca. 25.000 Drucke umfassenden Sammlung an „Familienpredigten" konnte die WLB bis jetzt über 2.700 Titel im OnlineKatalog verzeichnen und die Digitalisate online bereitstellen.

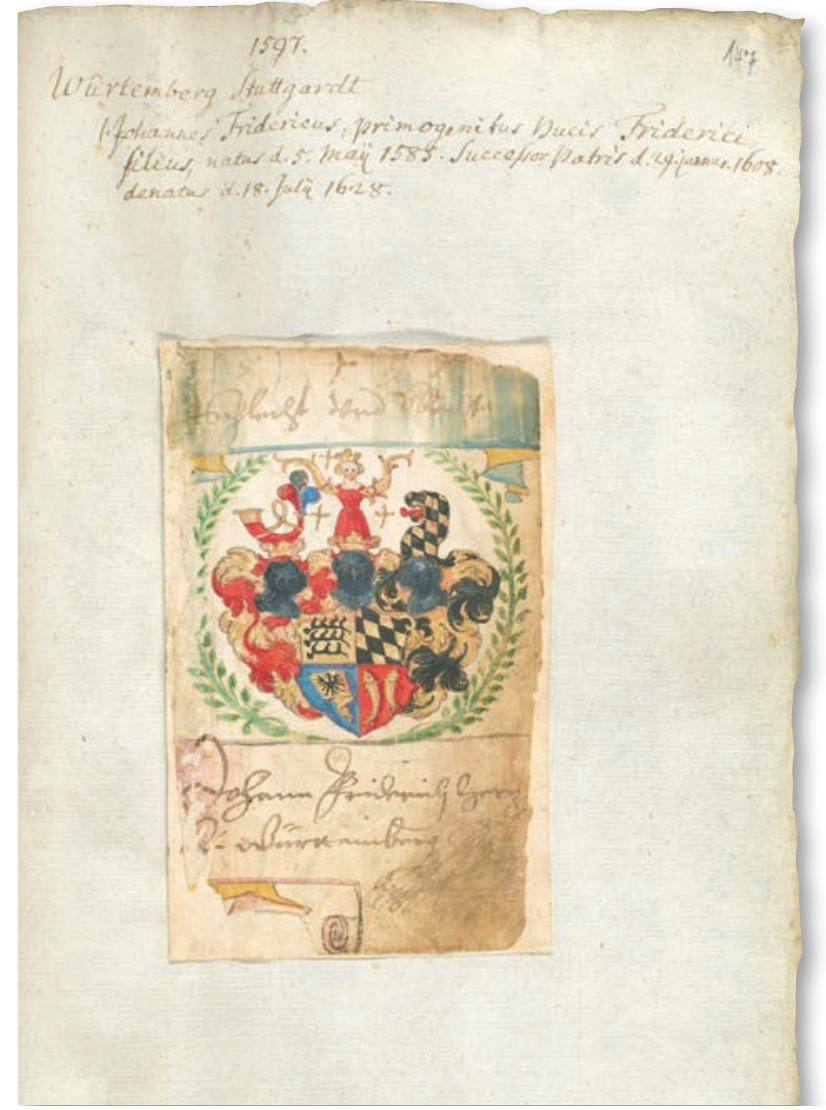

Abb. 4: Fürstliches Stamm-, Wappen- und Handschriften-Buch (Cod.hist. 2 906)

Die Digitalisierungsaktivitäten beziehen neben alten und wertvollen Drucken auch alle anderen historischen Sammlungen der WLB ein. Nach der Digitalisierung von Musikhandschriften konnte die Bearbeitung eines Teils der Sammlung des württembergischen Spitzenbeamten Friedrich Wilhelm Frommann (1707-1787) abgeschlossen werden, die Herzog Carl Eugen im Jahr 1785 für die damalige
Herzogliche Öffentliche Bibliothek erworben hatte. Einen besonders stark nachgefragten Bestand macht Frommanns umfangreiche, insgesamt 106 Alben umfassende Sammlung von Autographen und Wappen aus. Sie enthält neben ausgelösten Stammbuchblättern auch persönliche Briefe, Kanzleischreiben und andere handschriftliche Dokumente, die vornehmlich aus dem 16. bis beginnenden 18. Jahrhundert stammen. Darunter befinden sich z.B. 52 Bände mit Autographen bürgerlicher Personen (Cod. hist. $2^{\circ}$ 889) und eine „Wappen-Collection" von 36 Bänden mit adeligen und bürgerlichen Autographen und Wappen (Cod. hist. $2^{\circ} 888$ ).

Aus der Musiksammlung wurden Musikdrucke französischer Komponisten aus dem Vorbesitz der Deutschordenskommende Altshausen digitalisiert. Geplant ist, die Stuttgarter Theaterzettel der Jahre 1804-1945 elektronisch verfügbar zu machen.

Die Schefold-Sammlung mit über 2.600 gedruckten Ansichten württembergischer Ortschaften konnte vollständig digitalisiert werden. Kunst- wie wirtschaftshistorisch relevant sind die Handwerksbriefe der Sammlung Stopp, die mit Druckgraphiken zu den Wirkungsorten der Handwerker versehen wurden. Über 230 dieser Dokumente sind online.

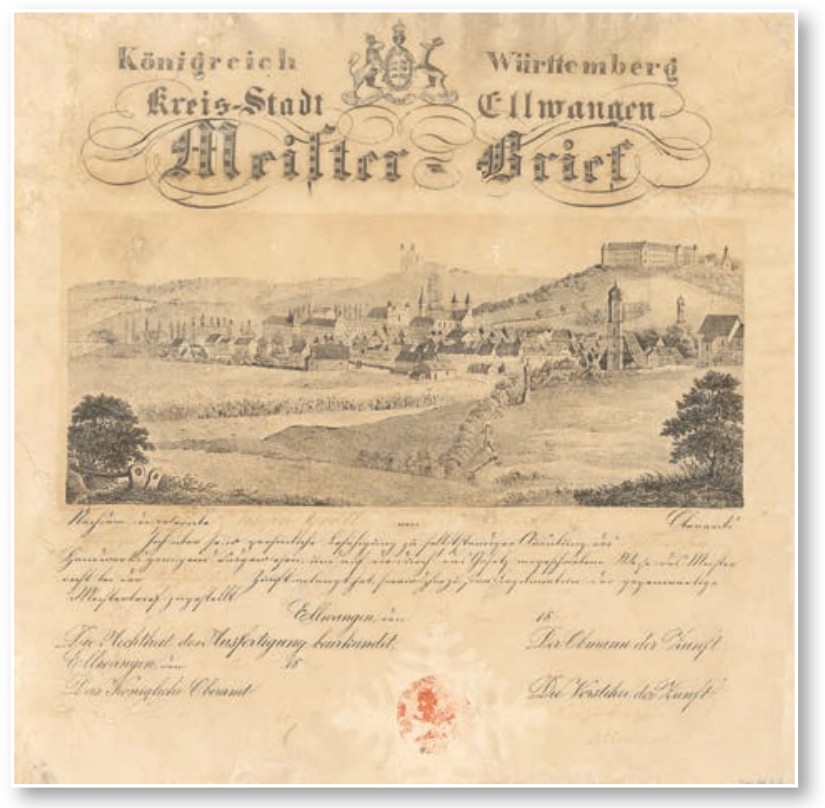

Abb. 5: Handwerksbrief aus Ellwangen, 1860 (Ans.fol.2-6)

Zu den Objekten neueren Druckdatums gehören die Stücke aus der Bibliothek für Zeitgeschichte (BfZ). Diese verfügt über eine umfangreiche Sammlung an Darstellungen zum Kriegserlebnis von Truppenteilen der deutschen Armee während 
des Ersten Weltkriegs. Die Soldaten identifizierten sich in hohem Maße insbesondere mit ihrem jeweiligen Regiment, das in Verbindung zu bestimmten

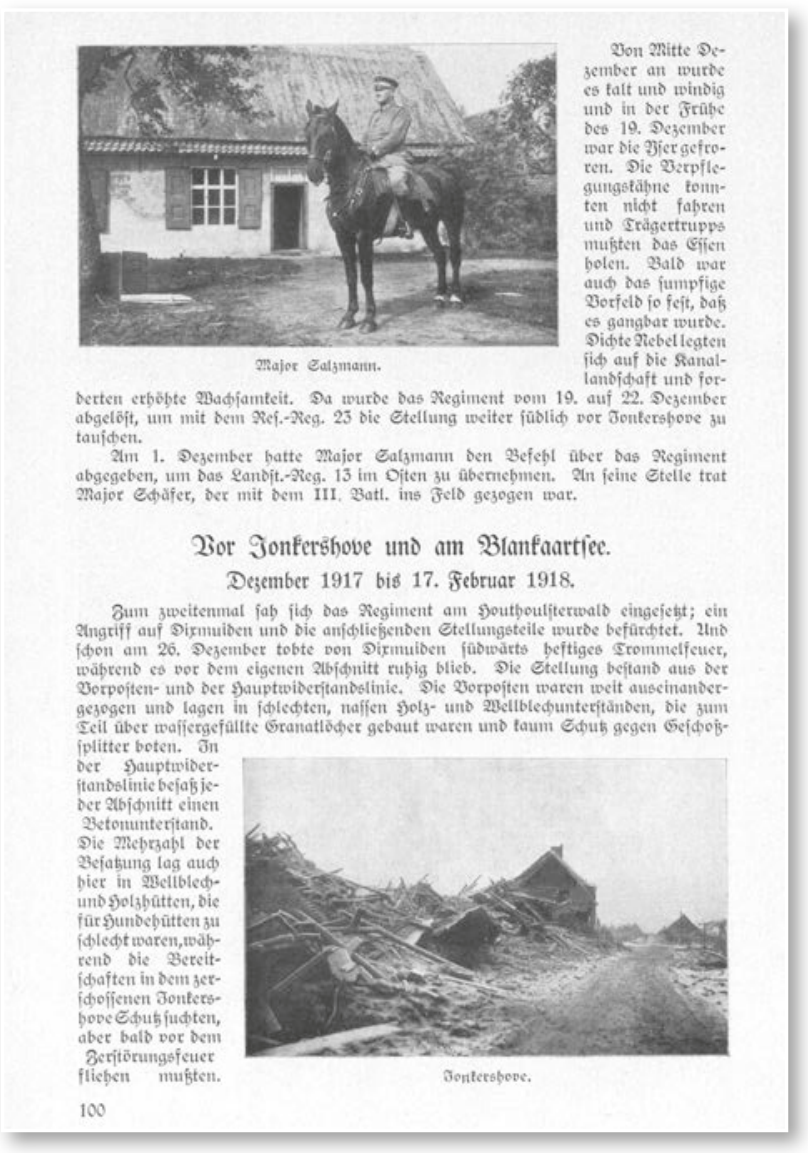

Abb. 6: Buch zur Regimentsgeschichte (F 396-7)

Orten bzw. Regionen stand. Die Regimentsgeschichten wurden häufig von Veteranen verfasst und dienten der Deutung des Krieges. Die Vergewisserung über gemeinsame Erlebnisse verknüpfte sich mit Patriotismus und Ehrung der Gefallenen. Die Regimentsgeschichten tragen in Verbindung mit Lebensdokumenten wie Feldpostbriefen und Tagebüchern in der Sammlung der BfZ dazu bei, die Quellenbasis für die Erforschung der Alltags-, Mentalitäts- und Kulturgeschichte des Krieges zu schaffen. Als Beispiel sei auf die Geschichte des Württembergischen Reserve-Infanterie-Regiments Nr. 119 im Ersten Weltkrieg hingewiesen (F 396-7). Zwei Fotos veranschaulichen die Geschicke dieses Regiments in Flandern. Vor dem Beginn der Kämpfe an der Brabantlinie im Winter 1917/1918 wurde der bisherige Regimentskommandeur an die zu diesem Zeitpunkt erfolgversprechendere Ostfront versetzt. Das idyllische Bild des Majors zu Pferde und die verwüstete flämische Ortschaft könnten kontrastreicher nicht sein.
Nach Klärungen zur bibliographischen Erfassung werden spätestens im Jahr 2020 auch die teilweise bereits digitalisierten Bände württembergischer Zeitschriften und Zeitungen (z.B. Riedlinger Zeitung) online verfügbar sein. In früheren Projekten aus Sondermitteln konnten u.a. die (baden-)württembergischen Landtagsprotokolle sowie die älteren Stuttgarter Adressbücher digitalisiert werden. Zu den technischen Verbesserungen seit dem letzten Bericht in dieser Zeitschrift (WLB-Forum

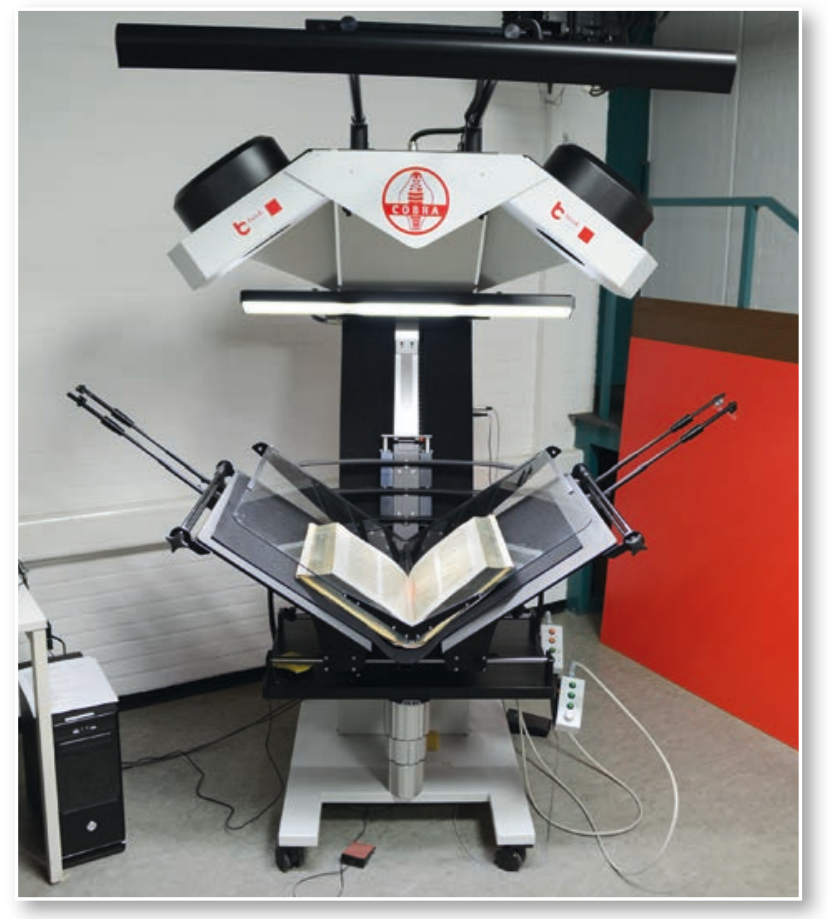

Abb. 7: Cobra High-End-Buchscanner

2017/1, S. 8-13) ist eine wichtige Erwerbung zu nennen. Die Ausstattung des Digitalisierungszentrums konnte 2018 durch einen book2net Cobra High-End-Buchscanner ergänzt werden. Dieser verfügt über zwei CMOS Systemkameras mit einer Auflösung von jeweils 71 Megapixeln. Mit den zwei Kameras können beide Seiten eines aufgeschlagenen Buches bis zu einer Größe von A2 gleichzeitig aufgenommen werden. Die doppelseitige Aufnahme gepaart mit der semirobotischen V-Glasandruck-Technologie erlaubt eine Digitalisierung mit sehr hoher Produktivität. Für die besonders schonende Behandlung von Vorlagen steht alternativ auch die motorische „Butterfly"-Andruckhilfe zur Verfügung.

\section{Christian Herrmann / Benjamin Mödinger}

